\title{
O câncer na perspectiva de cuidadores familiares e suas representações sociais
}

\section{The cancer from the perspective of Family caregivers and their social representations}

\author{
Silvio Eder Dias da Silva ${ }^{1}$, Esleane Vilela Vasconcelos ${ }^{2}$, Mary Elizabeth de Santana ${ }^{1}$, Jeferson Santos Araújo ${ }^{3}$
}

1. Docente da Faculdade de Enfermagem da Universidade Federal do Pará. Belém (PA) Brasil. 2. Secretaria de Estado de Saúde Pública do Pará. Belém (PA) Brasil. 3. Doutorando em Enfermagem Fundamental da Escola de Enfermagem de Ribeirão Preto, da Universidade de São Paulo. Ribeirão Preto (SP) Brasil.

\begin{abstract}
Resumo
Introdução: 0 câncer presente no indivíduo pode gerar diversas sensações na família, como medo, angústia e dor. O familiar cuidador em geral não está preparado para assumir tal dever, porém exerce um papel essencial na prática de cuidados ao paciente. Objetivo: A pesquisa objetivou identificar as representações sociais de cuidadores familiares de pacientes com câncer fora de possibilidades terapêuticas; e analisar suas implicações para o cuidado à saúde. Métodos: Trata-se de uma pesquisa descritiva com abordagem qualitativa. Foram entrevistados 30 cuidadores familiares que acompanhavam seus parentes internados na clínica de cuidados paliativos no Hospital Ophir Loyola, em Belém do Pará. Os dados foram obtidos por meio de uma entrevista semiestruturada e associação livre de palavras. Na análise do material, aplicou-se a análise temática. A pesquisa foi submetida ao Comitê de Ética da Escola de Enfermagem "Magalhães Barata" da Universidade do Estado do Pará sob protocolo CAAE-0048.0.321.000.11 de acordo com os preceitos da Resolução no 196/96/CNS/MS. Resultados: Da análise das entrevistas emergiram quatro temas: Câncer uma doença silenciosa; A prevenção do câncer por exames; O câncer no cotidiano do cuidador; A experiência do familiar com câncer influenciando a maneira do cuidador ver a doença: o cuidado com a saúde. Conclusão: 0 cuidado ao indivíduo em estado terminal foi apresentado como uma tarefa árdua e exaustiva pelos cuidadores. Logo, a Enfermagem tem papel relevante nessa relação com esses cuidadores, seja na realização de orientações, seja proporcionando-lhes um cuidado mais adequado.
\end{abstract}

Palavras-chave: Câncer. Cuidados Paliativos. Psicologia Social. Enfermagem.

\begin{abstract}
Introduction: The cancer which may occur in an individual can generate different sensations in the family, such as fear, anxiety and pain. Family caregivers in general are not prepared to assume this responsibility, but plays an essential role in the practice of patient care. Objective: The research aimed to identify the social representations of family caregivers of cancer patients out of therapeutic possibilities; and analyze its implications for health care. Methods: It is a descriptive research with a qualitative approach. They interviewed 30 family caregivers who accompanied their relatives admitted to the clinic of palliative care at the Hospital Ophir Loyola in Belém. Data were collected through semi-structured interviews and free association of words. In the material analysis applied to thematic analysis. The research was submitted to the Ethics Committee of the Nursing School "Barata Magalhães" State University of Pará under CAAE-0048.0.321.000.11 protocol in accordance with the provisions of Resolution No. 196/96 / CNS / MS. Results: The analysis of the interviews revealed four themes: cancer a silent disease; Cancer prevention examinations; The cancer caregiver's daily life; The experience of family cancer influencing the way the caregiver see the disease: the care of his health. Conclusion: The care to terminally ill individual was represented as a difficult and overwhelming task for caregivers. Therefore, nursing plays an important role in this relationship with these caregivers, whether in performing guidelines, is providing them with a more appropriate care.
\end{abstract}

Keywords: Cancer. Palliative care. Social Psychology. Nursing

\section{INTRODUÇÃO}

O câncer é uma doença preocupante em todo o mundo, ocasionado pelo crescimento desordenado de células que invadem tecidos e órgãos, sendo responsável por gerar lesões tumorais que se disseminam por todo o corpo, estas são chamadas metástases, cuja característica é carregar o selo definitivo da malignidade e sinal de mau prognóstico. Quando surgem metástases, quase sempre o tumor é incurável ${ }^{1}$.

O paciente que apresenta esse quadro de saúde passa a ser considerado um paciente fora de possibilidades terapêuticas, pois não responde mais a tratamentos curativos, necessitando de cuidados que resultem no máximo de conforto e bem estar, visando melhorar a qualidade de vida do paciente e sua família². Dependendo do grau de acometimento pela doença, os portadores de câncer avançado podem manifestar diversos déficits nas realizações das atividades de vida diária e comprometimento de necessidades humanas básicas. Dessa forma, o paciente pode possuir diversas dificuldades de cuidados de si necessitando, assim, de cuidados diferenciados para melhorar seu desempenho físico e cognitivo. Eis então que surge a figura do cuidador ${ }^{3}$.

A ocupação de cuidador integra a Classificação Brasileira de Ocupações - CBO sob o código 5162, que define o cuidador como alguém que cuida a partir dos objetivos estabelecidos por instituições especializadas ou responsáveis diretos, zelando pelo bem-estar do ser. É a pessoa que presta cuidados a outra pessoa que esteja necessitando, por estar acamada, com limitações físicas ou mentais, com ou sem remuneração. O cuidador familiar assume a responsabilidade pelas necessidades físicas e

Correspondência: Silvio Eder Dias da Silva. Professor Adjunto da Faculdade de Enfermagem da Universidade Federal do Pará (UFPA), Belém, Pará, Brasil. Trav. Angustura, 2932 - Apto. 1903, Torre B. Cep: 66093-040 Bairro do Marco. e-mail: silvioeder2003@yahoo.com.br

Conflito de interesse: Não há conflito de interesse por parte de qualquer um dos autores.

Recebido em: 07 Abril 2015; Revisado em: 6 Agosto 2015; Aceito em: 27 Agosto 2015. 
emocionais do outro que está incapacitado de se cuidar ${ }^{4}$.

Outro ponto é a importância de desvelar o universo do cuidador por meio da apreensão de suas representações sociais, a fim de compreender a verdadeira extensão da problemática, pois o conhecimento consensual propicia a compreensão de comportamentos e atitudes do grupo aqui pesquisado. Então, caracterizar as representações sociais dos cuidadores familiares propiciará a compreensão de como a patologia se insere em seu cotidiano, além de ser relevante sabermos que o câncer é um autêntico objeto psicossocial visto que o indivíduo capta as representações sociais e compartilha com seu grupo de pertença.

A enfermagem, nesse contexto, exerce um papel fundamental ao oferecer um cuidado direcionado as necessidades de cada paciente oncológico fora de possibilidades terapêuticas e sua família, de modo a ajudá-los a enfrentar da melhor maneira esse período crítico e a se preparem para o fim da vida e morte do ente querido ${ }^{5}$.

Este artigo segue os pressupostos da pesquisa social e, portanto, como objetivo identificar as representações sociais de cuidadores familiares de pacientes com câncer fora de possibilidades terapêuticas de cura e analisar suas implicações para o cuidado da saúde.

\section{METODOLOGIA}

Trata-se de pesquisa descritiva com abordagem qualitativa baseada preceitos da Teoria das Representações Sociais, cuja teoria busca a utilização de uma abordagem que observa como relevante o sujeito e sua relação com o ambiente no qual está inserido, tentando, assim uma compreensão das estruturas simbólicas dos cuidadores de pacientes com câncer avançado e suas representações frente às sociedades da qual fazem parte ${ }^{6}$.

As representações são fenômenos sociais que devem ser entendidos a partir do seu contexto de produção, ou seja, a partir das funções simbólicas e das formas de comunicação pelas quais circulam. Trata-se de conhecimentos que se compõem de elementos cognitivos socialmente elaborados e compartilhados, contribuindo para a construção de uma realidade comum e possibilitando a comunicação entre indivíduos.

Quanto ao método qualitativo, este permite compreender o homem diante dos fenômenos que os permeiam por meio de ferramentas que não visam mensurar seus achados, mas refletir com base no corpus do estudo, assim como validar os dados nos depoimentos e/ou documentos encontrados ${ }^{8}$. É neste momento em que a TRS se faz participante, pois a reflexão teórica sob o corpus permite o entendimento dos fenômenos a partir de uma lente específica, buscando responder às questões em estudo, que tratam do câncer sob a ótica dos cuidadores familiares.

A pesquisa se desenvolveu na clínica de cuidados paliativos do Hospital Ophir Loyola localizado em Belém do Pará, o qual é referência na região Norte no tratamento oncológico. Os participantes do estudo foram 30 cuidadores familiares que acompanhavam seus parentes internados, e que manifestaram disponibilidade e interesse em participar da pesquisa, após o conhecimento de seus objetivos e a assinatura do termo de consentimento livre e esclarecido.

Para coleta dos dados, foi utilizada a associação livre de palavras tendo as palavras indutoras "câncer" e "cuidar" de um parente com câncer seguido da expressão "por quê", permitindo a enunciação de significados e o conhecimento de práticas para posteriormente possibilitar a identificação dos processos de ancoragem e objetivação segundo a TRS $^{9}$. Também se realizou a aplicação da técnica de entrevista semiestruturada orientada por um roteiro.

$\mathrm{Na}$ análise do material coletado empregou-se a análise temática, cujo método é indicado para identificar, analisar e reportar padrões (aspectos comuns) entre os dados, além de organizar e descrever a série de dados em detalhes e interpretar os vários aspectos do objeto de pesquisa. Esse tipo de análise compõe seis fases: 1) Familiarização com os dados; 2) Geração de códigos; 3) Busca por temas; 4) Revisão contínua dos temas; 5) Definição dos temas, e; 6) Produção do relatório por meio de uma interpretação explicativa ${ }^{10}$.

Ressalta-se que o presente estudo foi aprovado pelo comitê de ética em pesquisa da Escola de Enfermagem Magalhães Barata da Universidade do Estado do Pará sob protocolo no CAAE 0048.0.321.000.11 e respeita todos os preceitos da Resolução 466/12 do Conselho Nacional de saúde, a qual regulariza e normatiza a pesquisa envolvendo seres humanos. Os sujeitos da pesquisa foram simbolizados alfanumericamente inviabilizando o reconhecimento de suas reais identidades.

\section{RESULTADOS}

Da análise das entrevistas emergiram quatro temas de acordo com as representações encontradas nos discursos dos participantes: Câncer uma doença silenciosa; A prevenção do câncer por exames; O câncer no cotidiano do cuidador; A experiência do familiar com câncer influenciando a maneira do cuidador ver a doença: o cuidado com a saúde.

\section{Câncer: Uma doença silenciosa}

Em muitos pacientes, uma das manifestações clínicas do câncer está relacionada com a evolução de metástases, mas inicialmente a doença em geral não apresenta sintomas significativos ${ }^{11}$. Sendo assim, observou-se que a ancoragem sobre a patologia se manifesta por meio de uma doença silenciosa como demonstram os relatos de doze entrevistados (40\%):

“Eu sei que é uma doença silenciosa. Às vezes 
quando chega a descobrir já é tarde e não dá pra tratar". (E3)

"O câncer é muito silencioso, se você não fizer exames ele já aparece avançado e aí não tem como tratar, é difícil". (E22)

Atrelando-se ao conhecimento consensual, o fenômeno câncer favoreceu a gênese de representações que o percebiam como uma doença silenciosa que tem que ser prevenida pelos indivíduos. Essas representações sociais possibilitaram a inserção do não familiar de se ter o câncer no cotidiano do cuidador? ${ }^{7}$.

Ainda no estudo, constatou-se que dezoito (60\%) dos cuidadores rotularam o câncer como uma doença que vai evoluindo de forma silenciosa que, se não for tratada precocemente, não oferece mais possibilidade de cura, como observado nos relatos:

“Eu sei que não tem cura. Só no início que sim. Se for descoberto no início, consegue cura. Mas se tiver avançado, não". (E8)

"Sei é que é uma doença que a pessoa não cura facilmente, só quando é descoberto no começo". (E14)

Prevalece nas falas dos depoentes a noção do câncer como doença incurável mas que, se tratado no início, pode ser curado. Além disso, nos discursos, acima a presença do conhecimento reificado favorecendo a gênese do conhecimento consensual por meio da ancoragem a ideia de morte. A partir deste contexto destaca-se que o câncer está vinculado ao imaginário social como uma doença incurável, sendo sua manifestação expressa nas comunicações dos depoentes como uma sentença de morte ${ }^{9}$.

A crença do câncer como uma doença silenciosa e incurável emergiu devido à sua força na memória cultural que é projetada sobre o tempo histórico e até mesmo sobre o presente e o futuro, modelados e transformados pelas diferentes formas de transmissão oral de uma geração para outra com sua mistura de crenças validadas socialmente, negociadas e manipuladas por meio das interações sociais da comunicação. Similar a outras culturas e formas sóciocognitivas do pensamento, sua função social é servir para desvelar a visão de mundo de um povo ou explicar uma prática, crença ou fenômeno natural ${ }^{12,13}$.

Outro meio difusor de representações sociais é a mídia. Para o autor, as representações sociais se modificam ou se atualizam dentro de relações de comunicação diferentes. Dessa forma, a mídia, integrada por um grupo de especialistas formadores e, sobretudo, difusores de representações sociais, é responsável pela estruturação de sistemas de comunicação que visam comunicar, difundir ou propagar determinadas representações. Dessa forma, os meios de comunicação de massa tornam-se um importante veículo de fortalecimento e difusão de ideologias específicas com grande poder de alcance ${ }^{14}$.

A maioria dos depoentes, dezessete cuidadores (57\%), referiu J. Health Biol Sci. 2015; 3(3):159-164 que aprendeu sobre o câncer na mídia:

"Vejo na televisão, no jornal as reportagens dizer isso". (E1)

"Em pesquisa na internet, em livros e revistas". (E21)

Também observamos, por meio dos relatos dos cuidadores entrevistados, que o significado do câncer ainda se encontra fortemente ancorado em morte, sofrimento, incapacidade e medo. Essas representações, como observamos, transitam na sociedade através das interações que se estabelecem cotidianamente pelo convívio com pessoas que assistiram as consequências trágicas da doença sejam por meio da morte de entes queridos ou por intermédio dos meios de comunicação social, a exemplo dos jornais que anunciam falecimentos de pessoas públicas vítimas de câncer. Tais informações passam a integrar o universo consensual, originando as representações vinculadas ao câncer ${ }^{14,15}$.

Nesse contexto, as representações sociais são fenômenos complexos ativados e em ação na vida social. Seu estudo torna possível o acesso a vários elementos, como informativos, cognitivos, ideológicos, normativos, crenças, valores, atitudes, opiniões e imagens. Esses elementos fornecem informações sobre os aspectos significantes da realidade social que, em relação com a ação, tornam-se o eixo das pesquisas científicas que buscam analisá-las ${ }^{16}$.

Nesta unidade, foi possível perceber como a memória cultural que passa de geração a geração foi primordial para que os depoentes atrelassem, em suas representações sociais, o câncer como uma doença silenciosa e incurável. Essas representações foram fundamentais para a gênese da segunda temática que será exposta a seguir.

\section{A prevenção do câncer por exames}

Nesta unidade, foi observado que os cuidadores relacionaram a prevenção do câncer com a realização de exames. Esta realidade está presente nas representações sociais dos cuidadores por intermédio da comunicação que foi primordial para a gênese dessas informações, conforme observadas nas falas de vinte e um cuidadores (70\%):

"Sim. Através de estar cuidando melhor do corpo e da saúde. Fazendo exames de rotina". (E7)

"Acredito que sim. A gente se prevenindo, se consultando. Então fazendo exames médicos anuais, para ver como está". (E10)

O câncer é uma doença que está relacionada ao estilo de vida e às exposições aos agentes ambientais. A maioria dos casos de câncer (80\%) está relacionada ao meio ambiente, onde encontramos um grande número de fatores de risco. Com a detecção precoce da doença em estágios iniciais, há melhores chances de cura. O exame clínico é importante; entretanto, cânceres mais internos necessitam de procedimento de imagem 
e testes especializados ${ }^{17}$.

Percebe-se nas falas dos cuidadores que a prevenção do câncer está ancorada na realização de exames como forma de rotina. Isso reflete o modelo biomédico da saúde por vivermos em uma sociedade regida pelo modelo cartesiano que preconiza a forma curativa de uma determinada doença. Isto é, o indivíduo tem que primeiramente adoecer para, a partir desse momento procurar os serviços de saúde. Esta visão biomédica não concebe a atitude de se procurar a assistência médica para ações preventivas, mas sim para o tratamento da doença ${ }^{18}$.

É importante elucidar que os sistemas de representações da doença estudada se constroem sempre a partir de um polo: a doença na terceira pessoa, isto é, a adesão de valores médicos de nossa época. É uma apreensão fundada sobre o conhecimento objetivo do sintoma e de sua etiologia, cujo corolário é a ocultação ou, pelo menos, um abandono ao segundo plano, da relação da doença com o psicológico e o social ${ }^{9}$.

Convém incluir esta cultura biomédica no campo da pesquisa antropológica, porque não há mais hoje em dia a representação da saúde que não se construa nesse contato e sob sua influência, mesmo que o doente proceda a uma triagem seletiva e uma reiteração do discurso especializado, e ainda que se recuse a inserir-se nos sistemas de medicalização oficiais ${ }^{18}$.

Esse modelo biomédico preconiza a realização de exames para o diagnóstico, porém não concebe que o indivíduo procure o serviço médico desprovido de sintomas; o ser dito "sadio" não necessita realizar exames. Assim, a maioria dos pacientes acometidos pelo câncer somente são diagnosticados quando a doença já se encontra em estado avançado. Como consequência, o atendimento primário desses pacientes ocorre já no serviço terciário, no caso da região norte no Hospital Ophir Loyola.

Nesta unidade, foi possível perceber que os depoentes atrelaram a prevenção da doença ao ato de fazer exames. Esse conhecimento consensual emergiu a partir do conhecimento reificado presente no modelo biomédico que preconiza fazer exames para esclarecer a sintomatologia sentida pelo indivíduo; porém, destaca-se que isso ocorreu nos pacientes dos cuidadores quando esses pacientes já se encontravam em estágio avançado da doença.

\section{O câncer no cotidiano do cuidador}

Os seres humanos se comunicam e interagem como forma de recriar uma realidade familiar com o propósito de apurar o significado do mundo, para torná-lo mais ordenado e para que as percepções reproduzam o mundo de uma maneira possível. Assim, as pessoas interpretam as situações vivenciadas, procurando dar sentido a elas ${ }^{19}$.

Dessa forma, as experiências de vida, no senso comum, são registradas na memória das pessoas. Algumas dessas são mais significativas e marcantes, talvez pelo fato de intensificarem emoções ou devido ao grau de implicação para a pessoa. 0 significado da situação vai depender das referências que se tem a seu respeito; portanto, um mesmo acontecimento pode ter interpretações diferentes. O meio social, através das relações cotidianas, influencia na construção da imagem para explicar os fenômenos percebidos, sendo esta determinante para seu enfrentamento ${ }^{20}$.

As situações vivenciadas que tiveram destaque no cotidiano dos cuidadores estão relacionadas com as complicações da doença, correspondendo a vinte e cinco (83\%) dos relatos:

"Foi quando ela chegou a vomitar sangue. Eu me sinto muito abalada quando lembro". (E12)

"Quando eu fiquei sentindo o odor. Pensei que a gente não ficava assim. Me sinto mal quando lembro". (E14)

Os sentimentos negativos apresentados apontam para uma representação carregada de muita emoção, decorrente, acima de tudo, do afeto pelo ente querido que vivencia uma situação de doença sem cura. O cotidiano dessa assistência é uma experiência complexa e muito sofrida que vai além da doença, pois irá acarretar mudanças de atitudes e comportamento do cuidador ${ }^{21}$

Vivenciar uma doença como o câncer está permeado de al-terações significativas no cotidiano de quem adoece e seus familiares. O câncer pode ser considerado cada vez mais um fenômeno não só biológico, mas também psicológico e social pelas várias situações de sofrimento imposta pela doença. Daí a necessidade da enfermagem também cuidar da família, a qual possui duplo papel, cuidadora e merecedora de cui-dados, sendo necessária uma atenção a todos os fatores e sentimentos que norteiam o cuidar para que a família possa repassar ao paciente um cuidado melhor e uma qualidade de vida mais digna a todos os envolvidos no processo de tratamento ${ }^{5,22}$.

Nesta unidade, os cuidadores relataram as situações marcantes que tiveram destaque no cotidiano do cuidado ao seu ente querido. As situações mais marcantes tiveram relação com as complicações do câncer e foram permeadas de sentimentos de dor e sofrimento. Essas experiências vivenciadas inseridas no cognitivo do indivíduo são compartilhadas com seu grupo de pertença, gerando assim, as práticas consensuais.

\section{A experiência do familiar com câncer influenciando a maneira do cuidador ver a doença: $O$ cuidado com a saúde}

Nesta unidade, os cuidadores relataram que o cotidiano de cuidados com o parente com câncer influenciou a maneira de cuidar da própria saúde; vinte e sete entrevistados (90\%) disseram que houve influência na maneira de cuidar de sua saúde, como observamos nos relatos abaixo:

"Influencia sim. Hoje eu penso em maneirar mais, não me sacrificar muito. Me cuidar mais para não passar o que ela está passando". (E3)

"Influencia muito. Eu procuro me cuidar mais, 
foi um alerta para eu me cuidar mais, ter mais atenção com a saúde". (E9)

Observamos, no estudo, que os cuidadores passaram a dar mais importância ao cuidado da saúde após a convivência com o parente acometido por câncer. Neste contexto, ficou claro que as representações sociais do câncer levaram os sujeitos a perceber a necessidade de conservarem sua própria saúde, passando o cuidado com a saúde a fazer parte da vida desses indivíduos, pois eles perceberam a importância de suas ações frente às medidas preventivas e mantenedoras da pessoa saudável.

O cuidador é uma pessoa que vivencia grande sofrimento, angústia e medo. O sofrimento apresenta-se por conviver com a doença do ser cuidado, a qual muitas vezes o consome; a angústia, por não conseguir reverter esse quadro, só podendo oferecer o cuidado que tem, e o medo, seja ele por não saber como proceder no cuidado, seja ele por perder o ser cuidado, interage em todos os momentos com o sentimento de perda e culpa ${ }^{18}$. A partir dessa vivência, ele irá incorporar a seu cognitivo, assim como repartir com seu grupo de pertença que deve ter mais atenção com sua saúde para não se acometido da mesma doença que seu ente querido.

Na busca de compreender o que representa o câncer em estágio avançado para esses cuidadores, encontramos símbolos e significados criados nos aspectos que os envolvem como prática social. A posição em que cada um se coloca, apresenta um conhecimento adquirido com seu mundo externo, por meio da comunicação com os seus. Dessa forma, a informação gerada entre os membros do grupo de cuidadores tem um papel fundamental no desenvolvimento do cuidado prestado e, como tal, revela a dimensão social das representações dos grupos acerca de um objeto; nesse caso, o cuidado de paciente com câncer avançado ${ }^{23}$.

Dessa forma, os conhecimentos utilizados pelo grupo de cuidadores são oriundos da comunicação formal e informal do canal social que os circunda, seja de seus familiares, amigos, vizinhança e da equipe de saúde a que tem acesso. Essas informações constroem e transformam os modos de pensar e agir, suas crenças e valores frente aos cuidados prestados ao indivíduo, de forma a guiar suas ações em face dos emanadores dos sentimentos de medo e morte, da velocidade em que 0 câncer se apresenta ${ }^{24}$.

As representações sociais de doença dos cuidadores sobre os seres a que prestam cuidados fazem que eles passem mais a valorizar seus próprios cuidados com a própria saúde. 0 conhecimento consensual os faz ter um olhar mais cuidadoso com sua saúde, como foi evidenciado anteriormente no ato de se submeter a exames de forma precoce para diagnosticar o câncer que acomete agora seu ente querido ${ }^{25}$.

Assim, nesta unidade foi identificado como o cuidador em suas representações sociais percebe que o seu ente querido não teve as devidas precauções com sua saúde e por isso foi acometido por câncer; assim, ele usa esse fato como incentivo para cuidar de sua própria saúde.

\section{CONCLUSÃO}

Este estudo evidenciou que as representações sociais de cuidadores familiares de pacientes fora de possibilidades terapêuticas sobre o câncer são marcadas pelo sofrimento de cuidar de um parente que requer cuidados paliativos, mas que as implicações dessas representações favoreceram um novo comportamento sobre o cuidado da saúde desses cuidadores.

Observou-se o quanto é necessário compreender as representações sociais desses cuidadores sobre a doença e sua ação no cuidado que permeia essa relação entre o cuidador e seu ente querido acometido por uma doença em estágio avançado, reconhecendo, dessa forma, sua complexidade. Essa compreensão permite proporcionar a elaboração de estratégias que possam contribuir para um cuidado de saúde eficiente e eficaz a este grupo social.

É importante o enfermeiro dar atenção às vivências não só do paciente, mas, também, do cuidador e compreender as dificuldades enfrentadas por ele nesse processo de cuidar, de modo que possa elaborar intervenções valorizando os aspectos físicos, emocionais, sociais, culturais, espirituais e éticos. Dessa forma, é importante o planejamento de intervenções de orientação, suporte e apoio para toda a família e principalmente para o cuidador familiar.

Entende-se que a enfermagem tem papel relevante nessa relação de cuidado, por meio da realização de orientações com esses cuidadores, seja para o cuidado direto ao paciente seja para o cuidado com a saúde do cuidador, sensibilizando-o quanto à importância do cuidado com sua saúde. Para isso, é fundamental que a enfermagem conheça essas representações sociais como forma de proporcionar um cuidado mais adequado a esses cuidadores, já que prestar cuidado é estar disponível a assistir o ser humano em sua totalidade.

\section{REFERÊNCIAS}

1. Glenn L, Robins HI. A História natural e a biologia do câncer. In: Pollock RE, Doroshow JH, Khayat, D, Nakao A, O'Sullivan, B. Manual de oncologia clínica da UICC. 8.ed. São Paulo: Fundação Oncocentro de São Paulo; 2006.

2. Barbosa SMM, Vallente MT, Okay, Y. Medicina Paliativa: a redefinição da experiência humana no processo de adoecer. Revista da Sociedade Brasileira para o estudo da dor, São Paulo, 2001; 3(2): 61-68.
3. Encarnação JF, Farinasso ALC. A família e o familiar cuidador de pacientes fora de possibilidades terapêuticas: uma revisão integrativa. Semina: Ciências Biológicas e da Saúde. 2014 Jan-Jun; 35(1): 137-148.

4. Fratezi FR, Gutierrez BAO. Cuidador familiar do idoso em cuidados paliativos: o processo de morrer no domicílio. Cienc Saude Coletiva. 2011 Jul; 16(7): 32413248. doi: http://dx.doi.org/10.1590/S1413-81232011000800023. 
5. Shirado A, Morita T, Akazawa T, Miyashita M, Sato K, Tsuneto S, et al. Both maintaining hope and preparing for death: effects of physicians' and nurses' behaviors from bereaved family members' perspectives. J Pain Symptom Manage. 2013; 45(5): 848-858. doi: 10.1016/j.jpainsymman.2012.05.014. PubMed PMID: 23159680.

6. Moscovici S. Representações sociais: investigações em psicologia social. 5. ed. Petrópolis RJ: Vozes; 2007.

7. Jodelet, D. Representações Sociais: um domínio em expansão. In D. Jodelet (Org.). As Representações Sociais (pp. 17-44). Rio de Janeiro: Eduerj. 2002.

8. Minayo MCS (org.). Pesquisa social: teoria, método e criatividade. 29. ed. Petrópolis: Vozes, 2010. (Coleção temas sociais).

9. Moscovici, S. Representações sociais: investigações em psicologia social. Rio de Janeiro: Vozes; 2011.

10. Braun V, Clarkc V. Using thematic analysis in psychology. Qualitative Research in Psychology. 2006; 3(2): 77-101. doi: 10.1191/1478088706qp063oa.

11. Machado RRS, Costa MC. Disponibilidade da informação para pacientes de câncer: a internet como ferramenta de visibilidade e construção de empoderamento. Cadernos do Tempo Presente. 2015 Mar-Abr; 19: 53-66.

12. Mok E, Lam WM, Chan LN, Lau KP, Ng JS, Chan KS. The meaning of hope from the perspective of Chinese advanced cancer patients in Hong Kong. Int J Palliat Nurs. 2010 Jun; 16(6): 298 - 305. PubMed PMID: 20925293.

13. Leite RA. Os sentidos dos cuidados paliativos oncológicos atribuídos pelo cuidador familiar. [Dissertação]. Ribeirão Preto (SP): Universidade de São Paulo; 2011.

14. Ribeiro RG, Kruse MHL. The woman body in review: the imperative of beauty. Texto contexto Enferm. 2014 Jan-Mar; 23(1): 101-8. doi: http://dx.doi. org/10.1590/S0104-07072014000100012

15. Queiroz AHAB, Souza AMA, Pontes RJS. Cuidado no final da vida: reflexões sobre a morte e o morrer. Scientia. 2013; 1(2): 255-263.
16. Spink MJP. O conceito de representação social na abordagem psicossocial. Cad. Saúde Públ. 1993 Jul-Set; 9(3): 300-308. doi: http://dx.doi.org/10.1590/ s0102-311X1993000300017.

17. Ministério da Saúde (Brasil). Manual de bases técnicas da oncologia - SIA/ SUS - Sistema de Informações Ambulatoriais. 17. ed. Brasília: Ministério da Saúde; 2014. p. 14.

18. Araújo JS, Silva SED, Santana ME, Conceição VM, Vasconcelos EV, Santos LMS. O conhecimento do cuidar nas representações sociais de cuidadores. Revista Tempus Actas de Saúde Coletiva. 2012; 6(3): 101-111.

19. Marková, I. Dialogicidade e representações sociais: as dinâmicas da mente. Petrópolis: Vozes; 2006.

20. Paredes EC, Jodelet D. Pensamento mítico e representações sociais. Cuiabá: EdUFMT/FAPEMAT/EdFUNI; 2009.

21. Bousso RS, Misko MD, Mendes-Castillo AM, Rossato LM. Family Management Style Framework and Its Use With Families Who Have a Child Undergoing Palliative Care at Home. J Fam Nurs. 2012 Feb; 18(1): 91-122. doi: 10.1177/1074840711427038. PubMed PMID: 22223493.

22. Dale MJ, Johnston B. An exploration of the concerns of patients with inoperable lung cancer Int J Palliat Nurs. 2011Jun; 17(6): 285 - 290. PubMed PMID: 21727886

23. Andrade CG, Costa CFG, Lopes MEL. Cuidados paliativos: a comunicação como estratégia de cuidado para o paciente em fase terminal. Cien saude coletiva. 2013; 18(9): 2523-2530. doi: http://dx.doi.org/10.1590/S141381232013000900006

24. Borges MM, Santos Junior RA. Comunicação na transição para os cuidados paliativos: artigo de revisão. Rev. bras. educ. med. 2014 Abr-Jun; 38(2): 275282. doi: http://dx.doi.org/10.1590/S0100-55022014000200015.

25. Jodelet, D. Loucuras e representações sociais. Petrópolis: Vozes; 2005

Como citar este artigo/How to cite this article:

Silva SED, Vasconcelos EV, Santana ME, Araújo JS. O câncer na perspectiva de cuidadores familiares e suas representações sociais. J Health Biol Sci. 2015 Jul-Set; 3(3):159-164. 